\title{
ON LOWER RADICAL TYPE CONSTRUCTIONS
}

\author{
GUY J. COOKE
}

(Received 20 January 2011)

\begin{abstract}
2010 Mathematics subject classification: primary 16 N80.
Keywords and phrases: radical class, semisimple class, lower radical construction.
\end{abstract}

This research is essentially an investigation into lower radical type construction and the consequences thereof. Dealing primarily in associative rings, the aim of this thesis is to construct—and investigate-a generalized lower radical type construction that encapsulates McDougall's base radical class construction [1] and Stewart's strict radical class construction [3].

Beginning with some preliminary results and a brief account of the lower radical (type) constructions in associative rings, we develop the basic concepts and notations used throughout the investigation. After determining a number of substructures suitable for use in our lower radical type construction (a $\rho$-radical class), we go on to examine some of the similarities, differences and properties of the $\rho$-radical class relative to those of the existing constructions.

In the lattice of all radical classes, we focus on the connections that both the base and strict radical constructions have respectively to two specific $\rho$-radical constructions. Moreover, we determine that all such $\rho$-radicals are contained in nontrivial radical classes, and subsequently position these against the so called concrete radicals in the lattice of all radical classes. We also demonstrate that the structure of a lattice of $\rho$-radical classes is partially dependent on the generating class, and thus not fixed in terms of construction.

In considering the dual construction to the $\rho$-radical class, we learn that unlike the $\rho$-radical construction always generating a radical class, the dual construction need not always generate a semisimple class. We consider some of the cases in which the dual is semisimple, and give a sufficient condition to determine the $\rho$-properties that do generate a $\rho$-semisimple class. Whilst on the subject of semisimple classes,

Thesis submitted to Central Queensland University, May 2009. Degree approved, April 2010. Supervisor: Dr Robert McDougall.

(C) 2011 Australian Mathematical Publishing Association Inc. 0004-9727/2011 \$16.00 
we generalize the notion of the strongly $\mathcal{R}$-semisimple ring, and explore when a class of such rings is a radical class.

The thesis concludes by generalizing the $\rho$-radical construction to suit any arbitrary category of group-based structures (GS-category) [2]. In doing so we validate the $\rho$ radical construction in structures such as groups, near-rings, nonassociative rings, any variety of rings or algebras, (Hausdorff) topological groups and rings, and vector value spaces to name just a few. This has certainly opened the door for further investigation, in these and possibly other branches of mathematics.

The simplicity and flexibility of the theory developed in this research provides an accessibility that may have wider applications in algebra.

\section{References}

[1] R. G. McDougall, 'A generalisation of the lower radical class', Bull. Aust. Math. Soc. 59 (1999), 139-146.

[2] B. J. Gardner, Radical Theory (Longman Scientific and Technical, Harlow, 1989).

[3] P. N. Stewart, 'Strict radical classes of associative rings', Proc. Amer. Math. Soc. 39 (1973), 273-278.

GUY J. COOKE, 25 Temby Ave, Kalamunda, 6076, Australia

e-mail: gcooke@live.com.au 